\title{
Sistemas de producción de tomates (Solanum lycopersicum M.) en Nicaragua: una caracterización en el municipio de Tisma, Masaya, Nicaragua
}

\section{Tomato production systems (Solanum lycopersicum M.) in Nicaragua: A characterization in the municipality of Tisma, Masaya, Nicaragua}

\section{Lizbeth Castillo Martínez ${ }^{1}$, Juan Carlos Morán Centeno², Gregorio Varela Ochoa $^{2}$}

${ }^{1}$ MSc en Sanidad Vegetal, ORCID: https://orcid.org/0000-0001-5371-4099, Formuladora Nicaragüense Hanon Talavera, S.A. (FORMUNICA), departamento de registró de plaguicidas, ing.lizbethcastillo@gmail.com / 2 ORCID: https://orcid.org/0000-0001-6135-7271, Universidad Nacional Agraria, Facultad de Agronomía, juan.moran@ci.una.edu.ni / 2 ORCID: https://orcid.org/0000-0001-5158-4999, Universidad Nacional Agraria, Facultad de Agronomía, gregorio.varela@ci.una.edu.ni

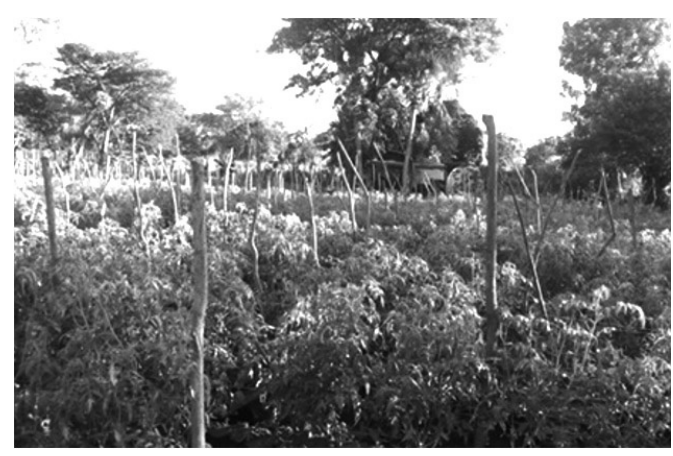

\section{RESUMEN}

Nicaragua es un país con condiciones climáticas apta para la agricultura, que permiten el establecimiento de diversas hortalizas de importancia en la seguridad alimentaria. El presente estudio se realizó en el municipio de Tisma, departamento de Masaya, en el año 2016, con el propósito de caracterizar los sistemas de producción del cultivo del Tomate (Solanum lycopersicum M.) a través de la generación de información. Se aplicó una encuesta socioeconómica en donde se incorporó el manejo agronómico y fitosanitario del cultivo y postcosecha de la producción. Basado en la información obtenida, se procedió a muestrear, procesar y analizar el registro de 25 Unidades de Producción (UP), las variables se sometieron a análisis univariados. Los resultados mostraron que en la mayoría de las UP predomina el sexo masculino, con edades promedios inferiores a los 40 años. Los productores son propietarios de sus UP, el nivel educativo determinó que $42.86 \%$ de los varones y $36.84 \%$ de las mujeres no lograron concluir la educación primaria. El $100 \%$ de los productores emplean semilla certificada de tomate, el manejo fitosanitario del cultivo se realizó empleando compuestos convencionales (Agroquímicos), recurriendo a la compra de productos selectivos, ya que el $96 \%$ reconocen las principales plagas y enfermedades que afectan este rubro, siendo la mosca blanca (Bemisia tabaci) la principal problemática, en la etapa de crecimiento del cultivo. En el manejo postcosecha un pequeño porcentaje desinfecta cajillas y medios de transporte, esto debido a que la producción tiene como destino el mercado local y es comercializado como hortaliza fresca.

Palabras clave: unidades de producción, manejo fitosanitario, manejo agronómico.

\section{ABSTRACT}

Nicaragua is a country with climatic conditions suitable for agriculture, which allow the establishment of various vegetables of importance in food security. The present study was carried out in the municipality of Tisma, department of Masaya, in 2016, with the purpose of characterizing the production systems of the tomato crop (Solanum lycopersicum M.) through the generation of information. A socioeconomic survey was applied where the agronomic and phytosanitary management of the crop and postharvest of the production was incorporated. Based on the information obtained, we proceeded to sample, process and analyze the record of 25 Production Units (UP), the variables were subjected to univariate analyzes. The results showed that in the majority of the UP the masculine sex predominates, with average ages less than 40 years. The producers are owners of their UP, the educational level determined that $42.86 \%$ of the men and $36.84 \%$ of the women did not manage to finish the primary education. $100 \%$ of the producers use certified tomato seed, the phytosanitary management of the crop was carried out using conventional compounds (Agrochemicals), resorting to the purchase of selective products since $96 \%$ recognize the main pests and diseases that affect this area, being the whitefly (Bemisia tabaci) the main problem, in the growth stage of the crop. In the postharvest handling a small percentage disinfects boxes and means of transport, this because the production is destined to the local market and is marketed as a fresh vegetable.

Keywords: Production units, phytosanitary management, agronomic management.
Recibido: 8 de junio del 2019

Aceptado: 26 de junio del 2019
Los artículos de la revista La Calera de la Universidad Nacional Agraria, Nicaragua, se comparten bajo términos de la licencia Creative Commons: Reconocimiento, No Comercial, Compartir Igual. Las autorizaciones adicionales a las aquí delimitadas se pueden obtener en el correo edgardo.jimenez@ci.una.edu.ni

C Copyright 2019. Universidad Nacional Agraria 


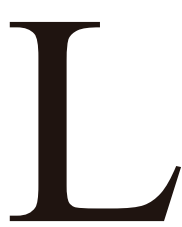

abrador y Altieri (2001), hacen referencia que el modelo agroindustrial se enfoca en la producción convencional en la producción de alimentos, sin importar las consecuencias ocasionadas al medio ambiente. Entre la problemática ocasionada por este modelo abarca el orden económico (endeudamiento de los agricultores, incremento de costos de producción), social (despoblamiento de áreas rurales, envejecimiento de la población rural) y ecológico (contaminación de recursos hídricos, erosión y pérdida de fertilidad de los suelos, deterioro de la biodiversidad, entre otros) que cada vez se hacen más frecuente (Casado y Mielgo, 2007).

El tomate (Solanum lycopersicum M) es la segunda hortaliza de mayor importancia económica a nivel mundial. En Centro América la superficie cultivada es de 21 000-25 000 hectáreas y el valor de la producción alcanza más de 50 millones de dólares con un rendimiento entre 15 a 20 ton ha ${ }^{-1}$. En Nicaragua se cultivan alrededor de 2469 hectáreas con un rendimiento promedio de 25.20 ton $\mathrm{ha}^{-1}$. Las zonas productoras de mayor importancia se encuentran en los departamentos de Matagalpa y Jinotega, particularmente en los Valles de Sébaco y Tomatoya y en menor escala en Estelí, Malacatoya, Tisma y Nandaime (Jiménez et al. 2018).

$\mathrm{Su}$ producción es un atractivo para los pequeños productores; utiliza mano de obra intensiva creando fuentes de ingresos en zonas rurales; así mismo estimula el empleo urbano (Laguna et al. 2004). Este rubro es afectado por una serie de problemas fitosanitarios a lo largo de su ciclo productivo, reduciendo significativamente los rendimientos y por ende causando pérdidas económicas. Las plagas insectiles, enfermedades y arvenses constituyen un factor limitante en la producción (Jones et al. 2001).

En el municipio de Tisma, Masaya, el tomate es la hortaliza de mayor importancia ya que genera una importate fuente de empleo e ingresos. Dicho municipio, presenta condiciones muy favorables para este cultivo (clima y topografía), cultivándose anualmente un área aproximada de 50 hectáreas, con rendimientos promedios de 750 cajas $\mathrm{mz}^{-1}$ (1 067 cajas ha $^{-1}$ ) (Rodríguez y Morales, 2007). Estableciéndose en forma de monocultivo, bajo un sistema de cielo abierto, lo que genera pérdidas de hasta un $50 \%$ del total de cosecha. En los últimos 10 años los pequeños productores de hortalizas de este municipio han controlado las plagas con productos químicos, lo que ha ocasionado efectos negativos en las poblaciones de artrópodos benéficos, en suelo, agua, salud humana y el ambiente (Hruska et al. 1997). Por lo que se efectuó el presente estudio con el objetivo de caracterizar los sistemas de producción del Tomate (Solanum lycopersicum M.) a través de la recopilación de información en el municipio de Tisma, Masaya, 2016. Esto para conocer las prácticas productivas que implementan los productores y la principal problemática a las que se enfrentan.

\section{MATERIALES Y MÉTODOS}

El estudio se llevó a cabo en el municipio de Tisma, departamento de Masaya, ubicado a 36 kilómetros de Managua, entre las coordenadas $12^{\circ} 04$ latitud Norte y $86^{\circ} 01$ longitud Oeste. Con una población de 12697 habitantes, altitud de $50 \mathrm{msnm}$, clima tropical de sabana y temperatura promedio de $27.5^{\circ} \mathrm{C}$ (AMUNIC, 2005). El comportamiento de las principales variables climáticas en el departamento de Masaya, fueron obtenidas en la estación meteorológica de Masaya en el período comprendido de enero a diciembre 2016. Los datos analizados correspondieron a temperatura, humedad relativa y precipitación. Se encontró que en los meses de septiembre y octubre las precipitaciones alcanzan los $250 \mathrm{~mm}$, con temperaturas de $29^{\circ} \mathrm{C}$. La humedad relativa presento un promedio de $85 \%$ (INETER, 2016).

El estudio fue no experimental del tipo cuantitativo, descriptivo. Consistió en visitas a las áreas de producción y productores claves. Mediante el método descriptivo se realizó una caracterización de los sistemas de producción de tomate, abarcando aspectos que definieron la situación social y de manejo productivo del rubro. En el municipio de Tisma, se encuentran reportados aproximadamente 455 productores de hortalizas (INIDE, 2011). De este universo 80 son productores de tomate, de los cuales fue seleccionada una muestra de 25 productores, siendo los criterios fundamentales la disponibilidad de participar en el estudio y producir tomate en sus parcelas para la aplicación de la encuesta y visita a las áreas de producción.

La metodología aplicada es un modelo para el desarrollo participativo. El estudio se dividió en tres fases las cuales se describen a continuación:

Primera fase: se definió la muestra a utilizar y las fincas, se seleccionó un máximo de 25 unidades productivas, se realizaron visitas a las instituciones del estado Instituto Nicaragüense de Tecnología Agropecuaria (INTA), Ministerio de Agricultura (MAG), Alcaldía en busca de información referente a la cantidad de productores de tomate en Tisma, de igual manera se buscó información en sitios de documentación.

La visita a informantes claves se efectuó para definir e identificar los productores y la elaboración y validación del instrumento de recopilación de la información en campo.

Segunda fase: Se recopiló información social y productiva de los productores referentes a la edad, composición familiar, nivel educativo y manejo del cultivo. Se ejecutaron las visitas a las fincas para constatar la información referente a las labores agrícolas implementadas en la producción (Cuadro 1).

Tercera fase: Se realizó el ordenamiento y procesamiento de la información procedente de las encuestas y visitas a las fincas. 
Para el cálculo de la muestra de datos socioeconómicos se utilizó la ecuación propuesta por Aguilar-Barojas (2005) en poblaciones finitas y variables categóricas.

$$
n=\frac{N Z^{2} p q}{d^{2}(N-1)+Z^{2} p q}
$$

Donde:

$\mathrm{n}=$ es el tamaño de la muestra.

$\mathrm{N}=$ tamaño de la población.

$\mathrm{Z}=$ valor de $\mathrm{Z}(\infty=0.1, \mathrm{Z}=1.88)$.

$\mathrm{p}=$ proporción aproximada del muestreo en el fenómeno en estudio de la población.

$\mathrm{q}=$ proporción de la población de referencia que no representa el fenómeno (1-p).

$\mathrm{E}=\mathrm{d}=$ Porcentaje de error asumido.
La información generada se organizó en bases de datos conformadas por variables categóricas y cuantitativas, se procesó en hojas electrónicas (Excel) y analizadas en SPSS v. 23 (IBM SPSS, 2010). Empleando estadística descriptiva (medidas de posición y tablas de contingencias).

\section{RESULTADOS Y DISCUSIÓN}

$\mathrm{El}$ análisis del componente social fue fundamental para conocer las condiciones de vida de la población, distribución de las edades, tenencia de la tierra y la tasa de analfabetismo presentado por la población.

Sexo y distribución de la población. El sexo del jefe de la Unidad de Producción (UP) es determinante al momento de tomar decisiones en los procesos productivos (Benavides y Morán, 2013). Dentro de las UP la influencia del sexo masculino es predominante, la Universidad Centro Americana

Cuadro 1. Descripción de las variables utilizadas en la caracterización de sistemas de producción de tomate en Tisma, Masaya

\begin{tabular}{|c|c|c|}
\hline Componente & Variables & Metodología empleada \\
\hline \multirow{8}{*}{$\begin{array}{l}\text { Social (Información } \\
\text { general de la unidad } \\
\text { de producción) }\end{array}$} & Edad (años): & Consultado a cada miembro que constituye la unidad de producción \\
\hline & Escolaridad: & Nivel académico de cada miembro de la familia \\
\hline & Sexo: & Preguntando el sexo de cada miembro de la familia \\
\hline & Ocupación: & Indagando mediante preguntas la ocupación de cada miembro de la familia. \\
\hline & Estado de la vivienda: & Mediante la observación directa de la vivienda del productor \\
\hline & $\begin{array}{l}\text { Acceso a los servicios } \\
\text { básicos (energía eléctri- } \\
\text { ca, agua potable, sanitar- } \\
\text { io): }\end{array}$ & $\begin{array}{l}\text { Indagando mediante preguntas el acceso a los servicios básicos por parte de la } \\
\text { familia }\end{array}$ \\
\hline & Tamaño de la finca: & Preguntando directamente al productor el tamaño de su unidad de producción \\
\hline & Tenencia de la tierra: & Consultando directamente al productor el estado legal de su unidad de producción \\
\hline \multirow{6}{*}{$\begin{array}{l}\text { Manejo Agronómico } \\
\text { del cultivo }\end{array}$} & Preparación del suelo: & Forma de preparación del suelo para la siembra \\
\hline & Época de siembra: & Época del año en que establece el cultivo \\
\hline & Variedad empleada & El material genético que emplea para la siembra \\
\hline & $\begin{array}{l}\text { Métodos de germinación } \\
\text { de semilla: }\end{array}$ & Método empleado por el productor para la germinación de la semilla \\
\hline & $\begin{array}{l}\text { Sustratos empleados en la } \\
\text { germinación: }\end{array}$ & $\begin{array}{l}\text { Indagando directamente con el productor el tipo de sustrato empleado para la ger- } \\
\text { minación de la semilla }\end{array}$ \\
\hline & Tipo de fertilización: & Tipo de fertilización empleado para la nutrición del cultivo \\
\hline \multirow[t]{3}{*}{$\begin{array}{l}\text { Manejo Fitosanitario } \\
\text { del cultivo }\end{array}$} & $\begin{array}{l}\text { Manejo de plagas y enfer- } \\
\text { medades: }\end{array}$ & El manejo fitosanitario en que se incurre para producir tomate \\
\hline & $\begin{array}{l}\text { Monitoreo de plagas y en- } \\
\text { fermedades: }\end{array}$ & Si realiza monitoreo de plagas y enfermedades en la unidad de producción \\
\hline & $\begin{array}{l}\text { Emplea prácticas para el } \\
\text { manejo de plagas y enfer- } \\
\text { medades: }\end{array}$ & $\begin{array}{l}\text { Si usa algún tipo de práctica para el manejo de plagas que afectan el cultivo del } \\
\text { tomate }\end{array}$ \\
\hline
\end{tabular}


(UCA, 2010), menciona que alrededor del $75 \%$ de los sistemas de producción cuentan con mayor presencia de personas del sexo masculino. En los sistemas de producción evaluados se contabilizaron 87 miembros, el sexo predominante fue el masculino que representan el $56.32 \%(\mathrm{n}=49)$, los restantes miembros correspondieron al sexo femenino con $43.68 \%$ $(\mathrm{n}=38)$. Esto difiere a lo reportado por el Delgadillo (2004), quien menciona que por cada 97.2 varones existen $100 \mathrm{mu}-$ jeres.

En las UP se encontró que las edades de los miembros de las familias productoras están en dependencia del sexo. El sexo masculino predominó en los rangos de 35-39 años y 50-54 años. En el caso del sexo femenino las edades varían hasta los 44 años, predominando los rangos de 30-34 y 20-24 años (Figura 1). Esto indica que los integrantes de las familias productoras son mayores a 20 años y menores a 60 años clasificándolos dentro de la población económicamente activa (PEA), siendo un recurso importante en los procesos productivos. Esto concuerda con lo publicado por el Delgadillo (2004), quien reporta que Nicaragua es un país que se encuentra en una etapa intermedia de la transición demográfica. CELADE (2004), indica que la población de Nicaragua presenta edades menores a los 15 años. Watkins (2008), reporta que en el 2005 el $37.9 \%$ y $4 \%$ correspondió a personas menores a 15 años y mayores a los 65 años, respectivamente en Nicaragua.
UNESCO (2013) manifiesta que existe una estrecha relación entre el nivel de educación con la demografía. Es importante señalar que entre los varones no existen analfabetas. El $42.86 \%$ no finalizó los estudios de primaria. El 30.61\% llegaron a concluir una formación superior (universidad completa). En cambio, el $36.84 \%$ de las mujeres no completaron los estudios de primaria y $21.05 \%$ la universidad completa. Para este sexo se encontró que un caso que no sabe leer ni escribir.

Característica de las viviendas. En las unidades de producción se determinó que el 56\% de las viviendas se encuentran catalogadas como buenas a muy buenas, el $84 \%$ presentan techo de zinc y $16 \%$ teja. Benavides y Morán (2013) encontraron que en el área rural de Nicaragua emplean materiales que fácilmente son adquiridos dentro de la comunidad o el municipio, estos mismos autores mencionan que debido a esto el principal material utilizado en los techos de los hogares es el zinc. Al analizar el material de construcción de las paredes el $84 \%$ son resistentes (Bloques $=48 \%$, Ladrillo $=8 \%$ y Cante$\mathrm{ra}=28 \%$ ). Con respeto al piso de las viviendas predomina el Ladrillo (60\%), concreto (20\%) y un $16 \%$ cuentan con piso de tierra. Basados en estos resultados posiblemente se podría asociar el estado de la vivienda con los ingresos obtenidos en la producción de tomate (Cuadro 2).

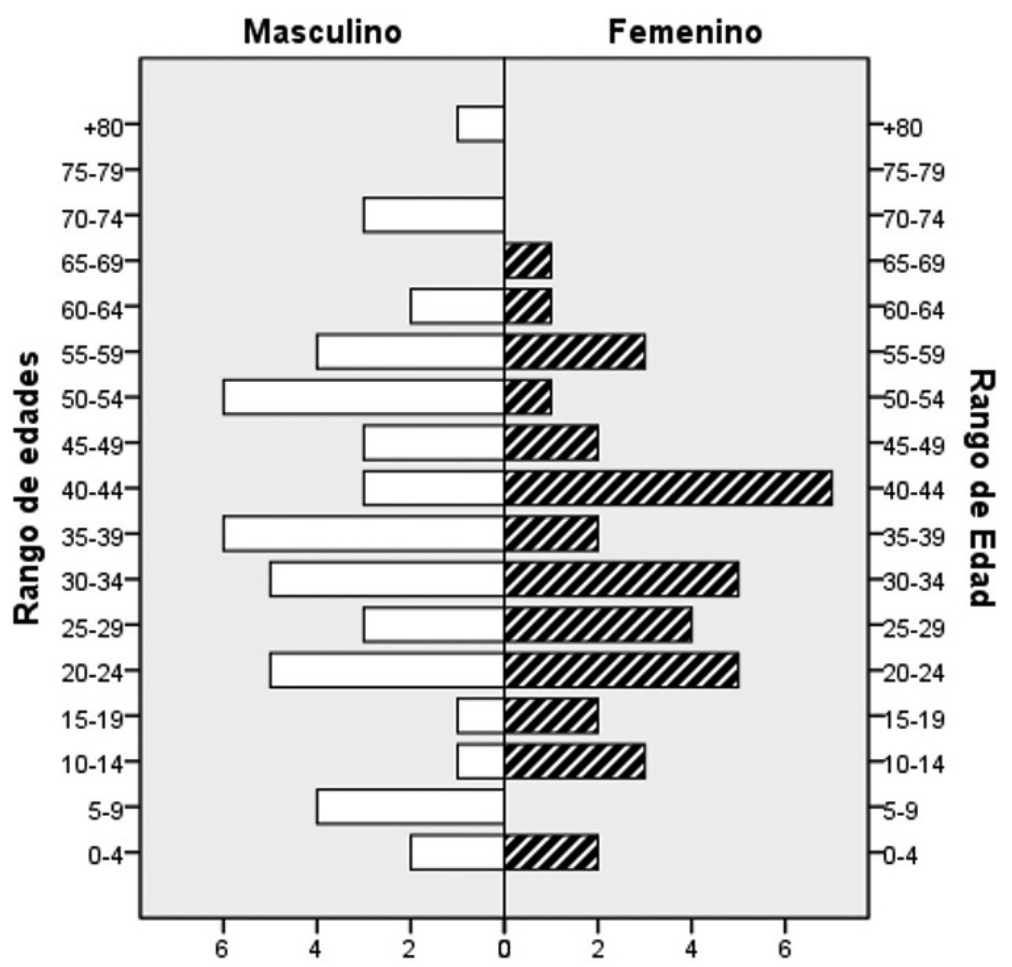

Figura 1. Pirámide poblacional según el sexo de los miembros que conforman las unidades de producción de tomate en el municipio de Tisma, Masaya (n=94 I IC=36 \pm 18.62 ). 
Cuadro 2. Características de la vivienda encontradas en las unidades de producción de tomate, Tisma, Masaya, 2016

\begin{tabular}{|c|c|c|c|c|c|c|c|c|c|c|c|}
\hline \multicolumn{3}{|c|}{ Piso } & \multicolumn{3}{|c|}{ Pared } & \multicolumn{3}{|c|}{ Techo } & \multicolumn{3}{|c|}{ Estado de la vivienda } \\
\hline Materiales & Frec & $\%$ & Materiales & Frec & $\%$ & Materiales & Frec & $\%$ & Estado & Frec & $\%$ \\
\hline Tierra & 4 & 16 & Madera & 4 & 16 & Zinc & 21 & 84 & Mala & 1 & 4 \\
\hline Ladrillo & 15 & 60 & Bloque & 12 & 48 & Teja & 4 & 16 & Regular & 10 & 40 \\
\hline Concreto & 5 & 20 & Ladrillo & 2 & 8 & & & & Buena & 10 & 40 \\
\hline Otros & 1 & 4 & Cantera & 7 & 28 & & & & Muy Buena & 4 & 16 \\
\hline Total & 25 & 100 & & 25 & 100 & & 25 & 100 & & 25 & 100 \\
\hline
\end{tabular}

Frec $=$ Frecuencia.

Tenencia de la UP. El derecho a la propiedad y tenencia de la tierra, encierra parte de un estado democrático en la sociedad nicaragüense; el cual debe estar gobernado por leyes acordadas entre todos, en el que se reconocen y respetan la libertad individual a la propiedad y la tenencia de la tierra (Espinoza y Castellón, 2015). El 52\% ( $\mathrm{n}=13)$ de UP son propietarios, $28 \%$ arrendatarios $(n=7)$ y el $20 \%$ prestan el terreno para cultivar $(\mathrm{n}=5)$. Entre los propietarios cuentan con muchos años de establecidos como productores de tomate. Los resultados obtenidos corresponden a los publicados por el INIDE (2011), siendo la tenencia un factor determinante en la mejora de las unidades de producción. En general se cuentan con promedio de 1.34 hectáreas, cultivada con tomate dentro del municipio.

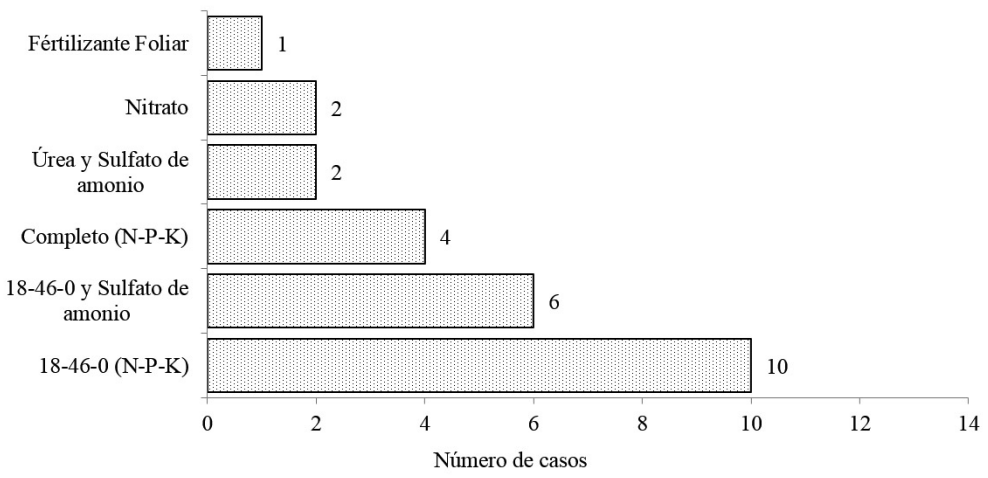

Figura 2. Productos empleados en la nutrición de las plántulas de tomate en el municipio de Tisma, Masaya.

Manejo de plántulas. En las UP, se utilizó la variedad de tomate Shanty, por su resistencia a la virosis, esto se debe a una alta presencia de mosca blanca (Bemisia tabaci) insecto vector de virus, que en los últimos años se ha convertido en la principal plaga en este rubro. Lo antes mencionado conlleva a que los productores adopten diferentes estrategias de manejo del cultivo, siendo el uso de semilleros la principal práctica implementada en las primeras fases de desarrollo de la planta, empleando sustrato estéril (KEKILA). El manejo de las plántulas de tomate, constituye una estrategia fundamental para garantizar el traslado de las mismas en óptimas condiciones al área de establecimiento (Úbeda y Meza, 2017). En el presente estudio se encontró que el principal método empleado en la germinación es mediante micro túneles $(96 \%)$ y semilleros $(4 \%)$. En cuanto a la fertilización empleada fue a base de productos químicos 18-46-0 (N-P-K), Sulfato de amonio, fertilizantes foliares entre otros. El control de arvenses se realizó de forma manual en el semillero y bandejas (Figura 2). Cuadras y García (2016), en estudio realizado en tomate indican que la nutrición de la plántula en el semillero brinda la oportunidad del productor de tener plantas vigorosas y sanas al momento de efectuar el trasplante al campo de siembra.
Manejo de arvenses en campo. El manejo de arvenses en el cultivo de tomate representó una práctica de mucha importancia, dado que compite con el cultivo de interés y ofrece refugio a insectos plagas que afectan el cultivo establecido. En cuanto a esta actividad el $44 \%(\mathrm{n}=11)$ emplean diferentes herbicidas (Yerbalade 15 EC: Fluazifop-p-butyl); sin embargo, el control mecánico aún se práctica en la plantación con $24 \%(n=6)$. El 48\% $(n=12)$ no respondió (NR/NA) sobre el mecanismo de control de arvense dentro del cultivo de tomate (Figura 3).

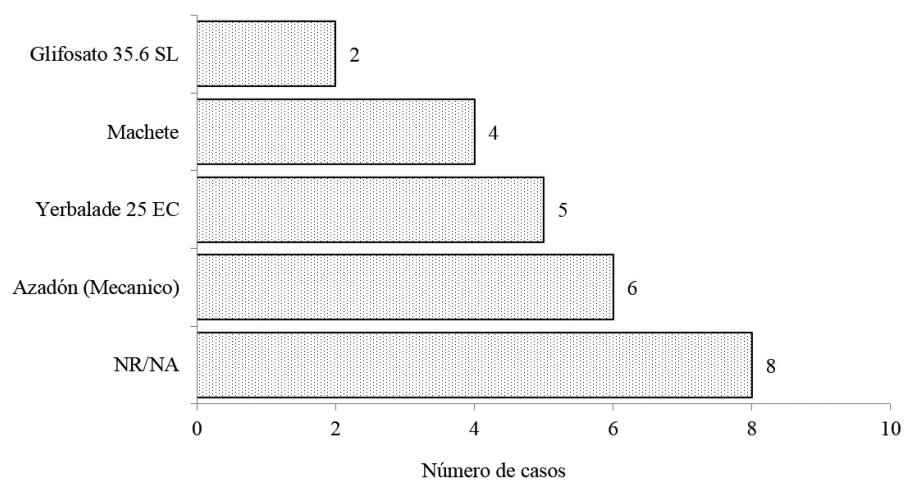

Figura 3. Manejo de arvenses en las unidades de producción en el municipio de Tisma, Masaya. 
Principales plagas y enfermedades que afectan el cultivo en campo. Holt et al. (2008), mencionan que la presencia de mosca blanca (Bemisia tabaci) en cantidades y tiempo después del trasplante es directamente proporcional al porcentaje de incidencia de virus en plantas. Se encontró que el 100\% de los productores tienen conocimiento de las plagas que afectan el cultivo (Cuadro 3). Para controlar estas afectaciones se han aplicado grandes cantidades de productos de origen convencional (Agroquímicos). En entrevistas efectuadas a los productores hacen mención que las afectaciones provocadas por estas plagas, reducen los rendimientos hasta en un $30 \%$ y si no se les da un manejo oportuno pueden llegar a causar pérdidas totales de la producción. Cerdas (2011) menciona que en los últimos años se ha incrementado la aplicación de productos convencionales en este municipio, para el manejo de plagas.

Cuadro 3. Principales plagas y enfermedades que afectan al cultivo del tomate,en el municipio de Tisma, Masaya $(\mathrm{n}=25)$

\begin{tabular}{ll}
\hline \multicolumn{1}{c}{ Plagas } & \multicolumn{1}{c}{ Nombre científico } \\
\hline Gallina ciega & Phyllophaga spp \\
Mosca blanca & Bemisia tabaci \\
Minador & Liriomyza sativae Blanchard \\
Trips & Frankliniella schultze \\
Áfidos & Aphis spp \\
Barrenador de fruto & Spodoptera spp \\
Ácaros & Polyphagotarsonemus latus \\
\hline \multicolumn{1}{c}{ Enfermedades } & \\
\hline Mal del talluelo & Fusarium sp, Pythium sp. \\
& Rizocthonia sp, Sclerotium sp \\
Mancha foliar & Fusarium oxysporum \\
Mancha bacteriana & $\begin{array}{l}\text { Pseudomonas solanacearum } \\
\text { Virus }\end{array}$ \\
Tizón temprano & Vector: Bemisia tabaci \\
\hline
\end{tabular}

Criterios de decisión sobre el uso de agroquímicos para el manejo de plagas y enfermedades. El productor de tomate decide la aplicación de los productos a utilizar para el control de plagas y enfermedades con el apoyo de las casas comerciales $32 \%(n=8)$, y la recomendación de un profesional en ciencias agropecuarias $32 \%(\mathrm{n}=8)$. Así mismo un $24 \%(\mathrm{n}=6)$ lo hace por tradición, y $4 \%(n=1)$, por recomendaciones de productores vecinos, rotación de agroquímicos (4\%), y presencia de plaga en campo (4\%). Los productores consultados expresaron que no cuentan con el conocimiento necesario, y se apoyan principalmente en las casas comerciales para tomar la decisión acerca del producto a usar, dosis y frecuencias de aplicación (Cuadro 4). Jiménez et al. (2012), manifiestan que en Tisma las fluctuaciones poblacionales de insectos plagas varían en el tiempo y la oportuna decisión del mecanismo de manejo por parte del productor es crucial para asegurar la producción. De estos el $68 \%(\mathrm{n}=17)$, emplean en la producción de tomate plaguicidas selectivos tales como Thiametoxam + Lambda cyhalothrin (Engeo 24.7 SC), Clorantraniliprole (Coragen 20 SC), el restante $(32 \%)$, aplican de amplio espectro como Cipermetrina (Cipermetrina 25 EC), Abamectina (Abamectina 1.8 EC), lo que conlleva a crear desbalance en el agro ecosistema.

Según Jiménez (2009), la aplicación de sustancias químicas es común en la producción de hortalizas, este autor menciona que la presencia y fluctuaciones de plagas conllevan a que los productores incrementen la cantidad de agroquímico en el ciclo del cultivo. Por otro lado, Cerda (2011) hace mención que en el municipio de Tisma el manejo convencional es predominante principalmente para el cultivo de tomate.

En el Cuadro 4 se representa el manejo de las plagas de suelo, en donde se emplea con mayor frecuencia Vydate 24 SL (25\%). Para controlar plagas de follaje se emplea Engeo $24.7 \mathrm{SC}(12 \%)$, y en la fase reproductiva el uso de Coragen $20 \mathrm{SC}(36 \%)$ es significativo. La aplicación de dichos productos conlleva a incrementar la contaminación ambiental, riesgo a la salud, resistencia y alteraciones al comportamiento alimenticio de los agentes causales de daños.

Cuadro 4. Principales productos químicos empleados en las unidades de producción en el suelo, follaje y frutos en el cultivo de tomate en el municipio de Tisma, Masaya

\begin{tabular}{|c|c|c|c|c|c|c|c|c|}
\hline \multicolumn{3}{|c|}{ Para plagas de suelo } & \multicolumn{3}{|c|}{ Para plagas de follaje } & \multicolumn{3}{|c|}{ Para plagas de fruto } \\
\hline Productos & Frec & $\%$ & Productos & Frec & $\%$ & Productos & Frec & $\%$ \\
\hline Lorsban $48 \mathrm{EC}$ & 1 & 4 & Actara & 2 & 8 & Coragen $20 \mathrm{SC}$ & 9 & 36 \\
\hline Cipermetrina 25EC & 1 & 4 & Confidor & 1 & 4 & Epingle $10 \mathrm{EW}$ & 1 & 4 \\
\hline Phyton 24 SA & 1 & 4 & Engeo $24.7 \mathrm{SC}$ & 3 & 12 & Otros & 15 & 60 \\
\hline Vydate 24 SL & 7 & 25 & Coragen $20 \mathrm{SC}$ & 2 & 8 & & & \\
\hline \multirow[t]{3}{*}{ Otros } & 18 & 64 & Epingle $10 \mathrm{EW}$ & 1 & 4 & & & \\
\hline & & & Tryclan 50 SP & 1 & 4 & & & \\
\hline & & & Otros & 15 & 60 & & & \\
\hline
\end{tabular}

Frec $=$ Frecuencia .

Manejo postcosecha. De acuerdo a Rodríguez y Morales, (2007), para la comercialización de frutas y hortalizas, tanto a nivel nacional como internacional, se han determinado estándares de calidad, que permitan una adecuada cadena de producción, comercialización y consumo. Los productores 
no efectúan medidas de desinfección de los materiales y herramientas utilizadas al momento de la cosecha, solo el $8 \%$ $(\mathrm{n}=2)$ desinfecta las cajillas, $16 \%(\mathrm{n}=4)$ aplican tratamiento al fruto y el $12 \%(n=3)$ desinfecta los medios de transporte (Cloro); estas prácticas no garantizan la inocuidad de los frutos de tomate al momento de ser comercializados en el mercado local.

\section{CONCLUSIÓN}

En los sistemas de producción de tomate los productores se dividen entre propietarios y arrendatarios con edades entre 35 y 54 años; el nivel de educación predominante es primaria, seguido de la formación profesional (Universitaria). Se encontró un único sistema productivo (Cielo abierto) y el uso de una sola variedad (Shanty), con manejo químico (convencional) para el control de plagas insectiles y enfermedades, con un nivel de tecnificación básica (riego por goteo y plásticultura). Las principales plagas que afectaron la producción son B. tabaci, Spodoptera spp, Ácaros, Fusarium sp, Pythium sp y Virus, reduciendo hasta en un $30 \%$ los rendimientos, siendo el principal destino de la producción el mercado departamental.

\section{AGRADECIMIENTOS}

A los productores de Tisma, Masaya por el apoyo brindado durante el levantamiento de la información en campo y su disponibilidad de compartir sus experiencias en la producción de tomate.

\section{REFERENCIAS BIBLIOGRÁFICAS}

Aguilar-Barojas, S. (2005). Fórmulas para el cálculo de la muestra en investigaciones de salud. Salud en Tabasco, 11(1-2), 333-338.

AMUNIC (Asociación de Municipios de Nicaragua). (2005). Municipios: Caracterización de municipios de Masaya (en línea). Managua, Nicaragua. Consultado el 09 de agosto. 2013. Disponible en http//www.amunic.org/

Benavides G. A y Morán-Centeno, J. C. (2013). Análisis numérico de características básicas de unidades familiares productivas (UFP) en nueve comunidades rurales de Nicaragua. La Calera, 13(21), 101-109.

CELADE (Centro Latinoamericano y Caribeño de Demografía). (2004). Boletín demográfico. Demographic Bulletin. América Latina y Caribe: Estimaciones y Proyecciones de Población 1950-2050. Latin America and Caribbean. Population Estimates and Projections. Año/ Year XXXVII, No. 73. Centro Latinoamericano y Caribeño de Demografía (CELADE) - División de Población Latin American and Caribbean Demographic Centre (CELADE) - Population Division. Santiago de Chile / Santiago, Chile. p. 252.

Cerda, C. John, K. (2011). Evaluación de alternativas de manejo contra el complejo mosca blanca (Bemisia tabaci Gennadius)-Geminivirus en el cultivo de tomate [Solanum lycopersicum L.) = (Lycopersicum esculentum Mill.)] en Tisma, Masaya (2009) y Camoapa, Boaco (2010 (Tesis de maestria). Universidad Nacional Agraria, Nicaragua.

Cuadra Aguilera, F., \& Garcia Ramos, D. U. (2016). Evaluación de tres láminas de riego por goteo y dosis de biofertilizante EM-5 (Sutocho) sobre el crecimiento, desarrollo y producción de tomate (Solanum lycopersicum Mill)) cv. Shanty, UNA, 2016 (Doctoral dissertation, Universidad Nacional Agraria).

Espinoza, E. M. M., \& Castellón, J. R. A. (2015). Tenencia de la tierra de acuerdo al IV CENAGRO de INIDE. REICE: Revista Electrónica de Investigación en Ciencias Económicas, 3(5), 140-162

Laguna, T., Pavon, J. F., Nicaragua Altamirano, K., \& Estrada Rizo, C. (2004). Manejo integrado de plagas: Guia MIP en el cultivo de la chiltoma. Ministerio de Agricultura y Ganaderia y Forestal, Managua (Nicaragua). Instituto Nicaraguense de Tecnologia Agropecuaria, Managua (Nicaragua).

Casado, G. G., \& Mielgo, A. A. (2007). La investigación participativa en agroecología: una herramienta para el desarrollo sustentable. Revista Ecosistemas, 16(1).

Holt, J., Pavis, C., Marquier, M., Chancellor, T. C. B., Urbino, C., \& Boissot, N. (2008). Insect-screened cultivation to reduce the invasion of tomato crops by Bemisia tabaci: modelling the impact on virus disease and vector. Agricultural and forest entomology, 10(1), 61-67.

Hruska, A. J., Vanegas, H. N., \& Perez, C. J. (1997). Analítico: resistencia de plagas agrícolas a insecticidas en Nicaragua: causas, situacion actual y manejo.

IBM SPSS Statistics 19 Command, Syntax Reference. Copyright C SPSS Inc. 1989. (2010). IBM SPSS Statistics versión 19. 2483 p.

INETER (Instituto Nicaragüense de Estudios Territoriales). (2008). Caracterización Climática del Departamento de Masaya. Managua, Nicaragua. $100 \mathrm{p}$.

Delgadillo Paguaga, N. (2004). Nicaragua: Estimaciones y proyecciones de poblacion nacional. Periodo 1950-2050. Instituto Nacional de Estadisticas y Censos, Managua (Nicaragua). CEPAL, Managua (Nicaragua). CELADE, Santiago (Chile)..

INIDE. (2011). Informe Final IV Censo Nacional Agropecuario. Managua.

Jiménez- Martínez, E; Chavarría, A., \& Rizo, Á. (2012). Manejo de mosca blanca (Bemisia tabaci Gennadius.) y geminivirus en semilleros de tomate (Lycopersicum esculentum Mill.) bajo protección física y química y su efecto en la producción. La Calera, 11(17), 05-13.

Jiménez, E. (2009). Métodos de control de plagas. Universidad Nacional Agraria. Managua, Nicaragua. 
Jones, J; Stall, R; Zitter, T. 2001. Plagas y enfermedades del tomate. The American Phytopathological Society. Mundi-Prensa. Madrid, ES. 74p.

Labrador, J., y Altieri, M. A. (2001). Agroecología y desarrollo. Aproximación a los fundamentos agroecológicos para la gestión sustentable de agrosistemas mediterráneos. Ed. Muindi Prensa-Universidad de Extremadura. Cácers-Madrid. ${ }^{\circ}$ Labrador, J.

UNESCO, F., EDUCATIVA, U. D. P., \& COMITE, E. P. D. (2013). Organización de las Naciones Unidas para la Educación, la Ciencia y la Cultura.

Jiménez-Martínez, E., Ríos-Peralta, H. T., \& Somarriba-Moncada, O. A. (2018). Evaluación de productos botánicos para manejo de mosca blanca (Bemisia tabaci Gennadius) y pulga del tomate (Halticu ssp) en el cultivo de tomate (Solanum lycopersicum Mill.), en Nicaragua. La Calera, 15(25), 63-69.

Watkins, K. (2008). Informe sobre Desarrollo Humano 2007-2008. La lucha contra el cambio climático: Solidaridad frente a un mundo dividido. DELOS: Desarrollo Local Sostenible, 1(1), 10.

Rodríguez, V. \& Morales, J. (2007). Evalución de alternativas de protección físico y químico de semilleros de tomate (Lycopersicum esculentum Mill) contra el ataque del complejo mosca blanca (Bemisia tabaci Gennandius)- geminivirus y su efecto en el rendimiento, en el municipio de Tisma, Masaya. Tesis Ing. ISPAF. Managua, Nicaragua. UNA. 62p.

Úbeda, R., Joaquín, E., \& Meza González, J. A. (2017). Evaluación de tres láminas de riego y tres distancias de siembra en el cultivo de tomate (Solanum lycopersicum L.) Cv. Butero, UNA, Managua, 2015 (Dissertation, Universidad Nacional Agraria).

Universidad Centroamericana (UCA). (2010). Problemática de la pobreza en Nicaragua. 27 pp. http://aulaweb.uca.edu.ni /blogs/edlacayo/files/2010/08/Pobreza-de-Nic.pdf. URL http://unesdoc.unesco.org/images /0013/001339/133942so.pdf 